\title{
¿Existe sarcopenia en pacientes menores de 30 años por criterio de bioimpedanciometría?
}

\author{
Is there sarcopenia in patients under 30 years by \\ bioelectrical impedance criteria?
}

\author{
Richard G. Buendía, Mónica E. Zambrano, Diana Gámez, \\ Nohora Reyes, luisa Fernanda Vásquez, Adelaida A. Reino, \\ Yein J. Morales, Laura Oyaga, Alejandra Morales \\ - Bogotá, D.C. (Colombia)
}

\section{Resumen}

La sarcopenia es una patología detectada principalmente en ancianos, se desconoce la prevalencia en personas jóvenes. Este estudio pretende determinar la prevalencia de sarcopenia en pacientes menores de 30 años mediante bioimpedanciometría y determinar sus factores asociados; en pacientes que acuden a consulta de endocrinología del Centro de Especialistas de Colsubsidio, Bogotá.

Metodología: estudio de corte transversal. Se estableció la correlación entre músculo corporal total y otros parámetros antropométricos como peso, talla, grasa corporal total, grasa visceral, sexo y edad. Determinó los factores asociados a sarcopenia mediante análisis multivariado.

Resultados: se incluyeron 501 pacientes, 315 mujeres (62.87\%) y 186 hombres $(37.13 \%)$ y se encontró una prevalencia de sarcopenia en pacientes menores de 30 años, clasificada como moderada $60.53 \%(n=46)$ y severa $22.37 \%(n=17) \mathrm{p}<0.001$; con $31.75 \%$ asociado a problemas de sobrepeso u obesidad $\mathrm{p}<0.001$. Además una correlación inversa entre el porcentaje de músculo total y edad, peso, grasa corporal total y grasa visceral tanto para mujeres y hombres respectivamente $\mathrm{p}<0.01$. Los factores independientes asociados a sarcopenia fue el sexo masculino OR $=1.09 \times 10^{16}$ (IC 95\% $\left.7.37 \times 10^{11}-1.62 \times 10^{20}\right) \mathrm{p}<0.001$; edad $\mathrm{OR}=1.15(\mathrm{IC} 95 \% 1.085-1.22) \mathrm{p}<0.001$ y grasa corporal total OR=1.9(IC 95\% 1.59-2.26) $\mathrm{p}<0.001$.

Conclusión: la definición de sarcopenia se enfoca en pacientes ancianos y es interesante cómo se aprecia pérdida de la masa muscular desde edades tempranas, asociados a problemas de sobrepeso u obesidad que podría corresponder a "obesidad sarcopénica". Consideramos que se debe realizar una definición de sarcopenia donde se incluya pacientes jóvenes. (Acta Med Colomb 2015; 40: 132-137).

Palabras clave: sarcopenia, adultos jóvenes, causas de sarcopenia, atrofia muscular, envejecimiento.

\footnotetext{
Abstract

Sarcopenia is a disease detected mainly in the elderly, and its prevalence in young people is unknown. This study aims to determine the prevalence of sarcopenia in patients under 30 years by bioelectrical impedance and determine its associated factors in patients attending endocrinology consultation of Specialists Center Colsubsidio Bogota.

Methodology: cross sectional study. The correlation between total body muscle and other anthropometric parameters such as weight, height, total body fat, visceral fat, sex and age was established. Factors associated with sarcopenia were determined by multivariate analysis.

Results: 501 patients, 315 women $(62.87 \%)$ and 186 men (37.13\%). Prevalence of sarcopenia found in patients under 30 years was classified as moderate in $60.53 \%(\mathrm{n}=46)$ and severe $22.37 \%$ $(\mathrm{n}=17) \mathrm{p}<0.001 ; 31.75 \%$ was associated with overweight or obesity $\mathrm{p}<0.001$. Besides, there was an inverse correlation between the percent of total muscle and age, weight, total body fat and visceral fat for both women and men respectively $\mathrm{p}<0.01$. Independent factors associated with sarcopenia were male gender OR $=1.09 \times 10^{16}\left(95 \%\right.$ CI $\left.7.37 \times 10^{11}-1,62 \times 10^{20}\right) \mathrm{p}<0.001$; age OR $=1.15(95 \%$ CI $1,085-1.22) \mathrm{p}<0.001$ and total body fat $\mathrm{OR}=1.9(95 \%$ CI $1.59-2,26) \mathrm{p}<0.001$.
}

Dr. Richard G. Buendía: Internista, Endocrinólogo y Epidemiólogo Clínico, Centro Médico de Especialistas Colsubsidio, Pontificia Universidad Javeriana; Dra. Mónica E. Zambrano: Ginecología y Endocrinología Ginecológica, Colsubsidio, Hospital Universitario de La Samaritana; Dra. Nohora Reyes: Internista; Dra. Luisa Fernanda Vásquez: Especialista en Medicina Familiar; Dras. Diana Gámez, Adelaida A. Reino, Yein J. Morales, Laura Oyaga y Alejandra Morales: Médicos Generales. Centro Médico de Especialistas Colsubsidio. Bogotá, D.C. (Colombia).

Correspondencia. Dr. Richard Buendía. Bogotá, D.C. (Colombia).

E-mail: Pernoll@gmail.com

Recibido: 1/VII/2014 Aceptado: 21/V/2015 
Conclusion: the definition of sarcopenia focuses on elderly patients, and it is interesting how loss of muscle mass can be seen from an early age, associated with overweight or obesity that might correspond to "sarcopenic obesity." We believe that a definition of sarcopenia where young patients be included should be made. (Acta Med Colomb 2015; 40: 132-137).

Keywords: sarcopenia, young adults, causes of sarcopenia, muscle athrophy, aging.

\section{Introducción}

La sarcopenia es una patología que se ha estudiado principalmente en pacientes ancianos, la cual ha tenido muchas definiciones y presenta dificultades en su medición (1). Además se asocia a mayor riesgo de caídas, fracturas (2) y aumento de la mortalidad (3).

Recientemente se dispone de la bioimpedanciometría, método seguro, que permite la medición de diferentes compartimentos corporales como es el músculo, grasa corporal total y grasa visceral; sin exponer al paciente a radiación y con una alta correlación con otros métodos como la resonancia magnética y la densitometría $(1,4-7)$. No hay estudios de pérdida de masa muscular compatible con la definición de sarcopenia en población menor de 30 años. Este estudio pretende determinar la prevalencia de sarcopenia, exclusivamente por criterio de bioimpedanciometría, como medición de masa muscular en pacientes menores de 30 años, que acuden a consulta de endocrinología en el Centro de Especialistas de Colsubsidio de la ciudad de Bogotá y como objetivo secundario determinar los factores asociados a la presencia de sarcopenia.

\section{Material y métodos}

Este es un estudio descriptivo de corte transversal, donde se busca determinar la proporción de pacientes con sarcopenia menores de 30 años, que acuden al servicio de endocrinología del Centro Médico de Especialistas de Colsubsidio.

Además establecer la correlación entre el porcentaje de músculo corporal total, con otros parámetros antropométricos como el peso, la talla, índice de masa corporal, grasa visceral, porcentaje grasa corporal total, incluyendo sexo y edad. Finalmente los factores asociados a sarcopenia mediante análisis multivariado usando regresión logística.

La medición de músculo corporal total se realizó por bioimpedanciometría. Se realizó en Bogotá, Colombia, con pacientes que acuden a consulta de endocrinología, provenientes de diferentes zonas del país. El protocolo fue sometido y aprobado por comité de ética local en Colsubsidio IPS. Se realizó consentimiento informado a todos los participantes y se siguieron los procedimientos éticos, según la Declaración de Helsinki.

El tamaño de la muestra se calculó con un nivel de significación de 5\%, una diferencia porcentual máxima esperada de $1 \%$, con una desviación estándar estimada de 0.05 y un coeficiente de concordancia y correlación mínimo de 0.90 con dos colas, estimando un tamaño de muestra de 366 pacientes.
Criterios de inclusión: pacientes mayores de 18 años de edad que acuden al servicio de consulta externa de endocrinología de la IPS Colsubsidio por cualquier causa, y que no presenten patología abdominal que pueda alterar la medición de grasa visceral.

Criterios de exclusión: pacientes con comorbilidades endocrinológicas que alteren la medición de grasa visceral, grasa total o masa muscular tales como: caquexia, hipotiroidismo fuera de metas en el año reciente, laparotomía reciente ( $\leq 8$ días), ascitis, embarazo, cáncer metastásico a cavidad abdominal, peritonitis, falla cardiaca (III - IV NYHA), masas intraabdominales, uso de marcapasos, visceromegalias, abscesos peritoneales, neumoperitoneo, obstrucción intestinal, megacolon tóxico y otras causas de distensión de asas, procedimientos invasivos a nivel abdominal (catéteres) y radioterapia.

Se incluyeron 501 pacientes, a los cuales se les aplicó un cuestionario con datos edad, sexo, peso, talla, índice de masa corporal, porcentaje de grasa visceral, grasa corporal total, porcentaje de músculo y medición de la circunferencia de cintura.

Se definió sarcopenia según criterios del consenso europeo de sarcopenia (8), el cual se basa en la medición del músculo en kilos /talla al cuadrado por bioimpedanciometría; no se toma el criterio de fuerza muscular, determinado por agarre para este estudio.

Esta definición dividido por sexo resulta: en hombres sarcopenia severa $\leq 8.50 \mathrm{Kg} / \mathrm{m}^{2}$, sarcopenia moderada $8.51-$ $10.75 \mathrm{Kg} / \mathrm{m}^{2}$ y normal $\geq 10.76 \mathrm{Kg} / \mathrm{m}^{2}$ y en mujeres sarcopenia severa $\leq 5.75 \mathrm{Kg} / \mathrm{m}^{2}$, sarcopenia moderada $5.76-6.75 \mathrm{Kg} / \mathrm{m}^{2}$ y normal $\geq 6.76 \mathrm{Kg} / \mathrm{m}^{2}$.

Para la bioimpedanciometría se utilizó el bioimpedanciómetro OMROM HBF-510w (9). Este impedanciómetro utiliza una corriente eléctrica de $50 \mathrm{kHz}$ y menos de 500 mcA. Se realiza medición de cuerpo entero (brazos a pies) en dos ocasiones. El impedanciómetro da información de índice de masa corporal, porcentaje de grasa visceral, grasa corporal total y porcentaje de músculo.

Para la medición del peso se usó DETECTO SCALE WEBB. MO.USA. con capacidad hasta 175 kilos y para la medición de talla se utilizó el tallímetro DETECTO WEBB. MO.USA. con capacidad de medir hasta dos metros.

Para el análisis descriptivo se usó promedios y desviaciones estándar para variables numéricas, con distribución normal y medianas con rango intercuartílico para variables numéricas sin distribución normal. 
Se realizaron pruebas de normalidad KolmogorovSmirnova para las variables numéricas y prueba de diferencia de varianzas.

Se usó la prueba U de Mann-Whitney para diferencia de medianas y $t$ de Student para diferencia de medias entre los grupos.

Se utilizó coeficiente de correlación de Spearman para determinación de la correlación entre porcentaje total de músculo y otras variables, con un intervalo de confianza de 95\%. Los valores de $\mathrm{p}$ se consideraron significativas menores a 0.05 a dos colas.

Para comparar las diferencias del porcentaje total de músculo por grupos etarios, se utilizó Anova no paramétrica Kruskal-wallis e imputación post-hoc con la prueba de Bonferroni.

Para la determinación de los factores asociados a sarcopenia (como una variable dicotómica), se realizó análisis multivariado por medio de regresión logística, ajustado por edad sexo, peso, porcentaje de grasa visceral, porcentaje de grasa corporal total con cálculo de bondad de ajuste de Hosmer-lemeshow, determinando el porcentaje de correctamente clasificados y finalmente el test de unión para determinar si el modelo contiene las variables suficientes e importantes del mismo.

Se uso STATA 12 (StataCorp) para realización de pruebas estadísticas.

Para el control de sesgos se seleccionaron los pacientes, tomando en cuenta la población en que va a utilizar la prueba en el futuro; el diseño del estudio es prospectivo con inclusión de pacientes de manera consecutiva y se controló la variabilidad interobservador, ya que todas las mediciones las realizó el mismo investigador.

\section{Resultados}

Se incluyeron 501 pacientes, 315 mujeres $(62.87 \%)$ y 186 hombres $(37.13 \%)$.

La Tabla 1 contiene las características de base de los pacientes incluidos.

Se realizaron pruebas de normalidad KolmogorovSmirnova para las variables numéricas y prueba de diferencia de varianzas, encontrando que la grasa corporal total es la única variable con distribución normal y homocedástica.

La mediana de la edad fue 50 años, tanto para hombres como para mujeres $(\mathrm{p}=0.39)$, el peso con una mediana 64.3 kilos para mujeres (rango 57.3-75) y 73.85 kilos para hombres (rango 67.3-83) $(\mathrm{p}<0.001)$; la mediana del índice de masa corporal fue 26.5 para mujeres y 26.4 para hombres $(\mathrm{p}=0.15)$; el porcentaje de músculo la mediana hallada fue $24.9 \%$ para mujeres y $34.45 \%$ para hombres $)(p<0.001)$. El porcentaje de grasa visceral fue mediana de $8 \%$ (rango 6-10) y mediana $11 \%$ (rango $8-14)(\mathrm{p}<0.001)$ (Tabla 1$)$.

Se encontró una correlación inversa robusta entre el porcentaje de músculo total y el porcentaje de grasa corporal total $(\mathrm{rho}=-0.855$ para mujeres y $\mathrm{rho}=-0.882$ para hombres $\mathrm{p}<0.01)$; así mismo, con grasa visceral y perímetro de cintura (Tabla 2).

Por grupos etáreos se encontraron diferencias en el porcentaje de músculo total, (anova no paramétrica Kruskal Wallis $\mathrm{p}<0.001$ ), tomando como referencia la edad entre 18 y 30 años, se evidencia pérdida significativa del porcentaje de músculo por cada década de la vida (Post-hoc Bonferroni), siendo más significativa entre 51-70 años (Tabla 3).

La prevalencia de sarcopenia en la población total estudiada $69.26 \%$ ( $n=347$ pacientes $)$, siendo $52.4 \%$ en hombres y $47.55 \%$ en mujeres $(p<0.001)$. Al clasificar la sarcopenia

Tabla 1. Características de base de la población estudiada $N=501$

\begin{tabular}{|c|c|c|c|}
\hline Variables & $\begin{array}{c}\text { n (\%) } \\
315(62.87)\end{array}$ & $\begin{array}{c}n(\%) \\
186(37.13)\end{array}$ & $\mathbf{p}$ \\
\hline Edad mediana (rango intercuatílico) & $\begin{array}{c}50 \\
(37-60)\end{array}$ & $\begin{array}{c}50.5 \\
(38-63)\end{array}$ & 0.399 \\
\hline Peso mediana (rango intercuatílico) & $\begin{array}{c}64.30 \\
(57.3-75)\end{array}$ & $\begin{array}{c}73.85 \\
(67.3-83)\end{array}$ & 0.001 \\
\hline Talla mediana (rango intercuatílico) & $\begin{array}{c}1.55 \\
(1.51-1.59)\end{array}$ & $\begin{array}{c}1.69 \\
(1.65-1.74)\end{array}$ & 0.001 \\
\hline Índice de masa corporal mediana (rango intercuatílico) & $\begin{array}{c}26.59 \\
(23.75-31.1)\end{array}$ & $\begin{array}{c}26.44 \\
(23.8-29.03)\end{array}$ & 0.15 \\
\hline Porcentaje grasa corporal total [media (sd)] & $\begin{array}{l}40.14 \\
(7.66)\end{array}$ & $\begin{array}{l}24.71 \\
(6.93)\end{array}$ & 0.001 \\
\hline Porcentaje de grasa visceral mediana (rango intercuatílico) & $\begin{array}{c}8 \\
(6-10)\end{array}$ & $\begin{array}{c}11 \\
(8-14)\end{array}$ & 0.001 \\
\hline Porcentaje de músculo mediana (rango intercuatílico) & $\begin{array}{c}24.9 \\
(22.8-26.8)\end{array}$ & $\begin{array}{c}34.45 \\
(32-37.1)\end{array}$ & 0.001 \\
\hline Perímetro de cintura mediana(rango intercuatílico) & $\begin{array}{c}87 \\
(78-97)\end{array}$ & $\begin{array}{c}92 \\
(87-100)\end{array}$ & 0.001 \\
\hline Músculo corporal total $\left(\mathrm{Kg} / \mathrm{m}^{2}\right)$ Mediana (rango intercuatílico) & $\begin{array}{c}6.69 \\
(5-9.81)\end{array}$ & $\begin{array}{c}9.09 \\
(6.57-11.77)\end{array}$ & 0.001 \\
\hline
\end{tabular}


TRABAJOS ORIGINALES • ¿Bioimpedanciometría para diagnóstico de sarcopenia en menores de 30 años?

Tabla 2. Correlación de porcentaje de músculo corporal total con otras variables antropométricas.

\begin{tabular}{|l|c|c|c|c|}
\hline Correlación Spearman (rho) & Mujer & $\mathbf{p}$ & Hombre & $\mathbf{p}$ \\
\hline Porcentaje de Músculo & & & & \\
Edad & -0.222 & 0.001 & -0.554 & 0.001 \\
\hline Peso & -0.470 & 0.001 & -0.642 & 0.001 \\
\hline Talla & 0.274 & 0.001 & 0.171 & 0.001 \\
\hline Índice de masa corporal & -0.626 & 0.001 & -0.762 & 0.001 \\
\hline Porcentaje de grasa corporal total & -0.855 & 0.001 & -0.882 & 0.001 \\
\hline Grasa visceral & -0.615 & 0.001 & -0.836 & 0.001 \\
\hline Perímetro de cintura & -0.489 & 0.001 & -0.793 & 0.001 \\
\hline
\end{tabular}

por grupo etario y por severidad según la clasificación del consenso europeo (8), el más frecuente fue el grado moderado de sarcopenia $51.7 \%$, seguido del grado severo de $17.56 \%$. En mayores de 70 años predomina el grado severo de osteopenia $53.85 \%$ de los pacientes de este grupo. Llama la atención la presencia de sarcopenia moderada entre la edad de 18 y 30 años, $60.5 \%$ de los pacientes de este grupo, $(\mathrm{p}<0.001)$ (Tabla 4).

Se encontró que en los pacientes sarcopénicos que corresponden a $54.18 \%(n=188)$ existe una asociación con problemas de sobrepeso u obesidad, así por grupos etáreos: menores de 30 años $31.75 \%(n=20), 31-40$ años $43.4 \%$ $(n=23), 41-50$ años $56.25 \%(n=36), 51-60$ años $64.47 \%$
Tabla 3. Diferencias en el porcentaje de músculo corporal total por grupos etáreos.

\begin{tabular}{|l|c|c|}
\hline Edad & $\begin{array}{c}\text { Pérdida de músculo corporal total } \\
\text { (porcentaje) }\end{array}$ & p \\
\hline 18 a 30 años & referencia & referencia \\
\hline 31- 40 años & -2.98 & 0.072 \\
\hline $41-50$ años & -2.40 & 0.180 \\
\hline 51-60 años & -4.39 & $<0.01$ \\
\hline 61-70 años & -4.59 & $<0.01$ \\
\hline Mayor 70 años & -1.95 & 0.819 \\
\hline
\end{tabular}

$(n=49), 61-70$ años $72.06 \%(n=49)$ y mayores de 70 años $47.83 \%(\mathrm{n}=11),(\mathrm{p}<0.001)$.

Para determinar los factores asociados a sarcopenia se realizó análisis univariado, encontrando que el factor que tienen mayor fuerza de asociación es el sexo masculino (OR: 41.36 intervalo de confianza IC 95\% 4.99-114.1 p<0.001) (Tabla 5).

Al realizar el análisis multivariado ajustado por edad sexo, peso, porcentaje de grasa visceral, porcentaje de grasa corporal total, se encuentra cómo la edad es un factor asociado a sarcopenia OR 1.15 IC95\% (1.085-1.22) (p<0.001); así mismo, el sexo masculino sigue siendo un factor robusto asociado a sarcopenia y el porcentaje de grasa corporal total (Tabla 6). Bondad de ajuste de Hosmer-Lemeshow $\mathrm{p}=0.11$, correctamente clasificados $91.82 \%$ y test de unión hat $\mathrm{p}<0.001$, hatsq $\mathrm{p}=0.063$.

Tabla 4. Grado de sarcopenia por grupos etarios.

\begin{tabular}{|l|c|c|c|}
\hline Edad intervalos & Severo & Moderado & Total \\
\hline 18 a 30 años & $17(22.37 \%)$ & $46(60.53 \%)$ & $13(17.11 \%)$ \\
\hline 31 a 40 años & $11(14.29 \%)$ & $42(54.55 \%)$ & $24(31.17 \%)$ \\
\hline 41 a 50 años & $11(10.48 \%)$ & $53(50.48 \%)$ & $41(39.05 \%)$ \\
\hline 51 a 60 años & $20(17.24 \%)$ & $56(48.28 \%)$ & $40(34.48 \%)$ \\
\hline 61 a 70 años & $15(14.85 \%)$ & $53(52.48 \%)$ & $33(32.67 \%)$ \\
\hline Mayor de 70 años & $14(53.85 \%)$ & $9(34.62 \%)$ & $3(11.54 \%)$ \\
\hline Total & $\mathbf{8 8}(\mathbf{1 7 . 5 6 \% )}$ & $\mathbf{2 5 9}(\mathbf{5 1 . 7 \%})$ & $\mathbf{1 0 1}$ \\
\hline
\end{tabular}

Tabla 5. Factores asociados a sarcopenia análisis bivariado.

\begin{tabular}{|l|c|c|c|c|}
\hline Factor & OR & \multicolumn{2}{|c|}{$\begin{array}{c}\text { Intervalo de confianza } \\
95 \%\end{array}$} & p \\
\hline Edad & 0.99 & 0.98 & 1.01 & 0.283 \\
\hline Sexo masculino & 41.36 & 4.99 & 114.15 & $<0.001$ \\
\hline Peso & 0.95 & 0.95 & 0.97 & $<0.001$ \\
\hline IMC & 0.76 & 0.72 & 0.80 & $<0.001$ \\
\hline Grasa total & 0.86 & 0.84 & 0.89 & $<0.001$ \\
\hline Grasa visceral & 0.94 & 0.91 & 0.99 & 0.018 \\
\hline
\end{tabular}

Tabla 6. Factores asociados a sarcopenia análisis multivariado.

\begin{tabular}{|l|c|c|c|c|}
\hline & OR & \multicolumn{2}{|c|}{$\begin{array}{c}\text { Intervalo de confianza } \\
\mathbf{9 5 \%}\end{array}$} & p \\
\hline Edad & 1.15 & 1.085 & 1.22 & $<0.001$ \\
\hline Sexo Masculino & $1.09 \times 10^{16}$ & $7.37 \times 10^{11}$ & $1.62 \times 10^{20}$ & $<0.001$ \\
\hline Peso & 0.77 & 0.72 & 0.83 & $<0.001$ \\
\hline Grasa total & 1.90 & 1.59 & 2.26 & $<0.001$ \\
\hline $\begin{array}{l}\text { Ajustado por edad sexo, peso, porcentaje de grasa visceral, porcentaje de grasa corporal } \\
\text { total, bondad de ajuste de Hosmer-Lemeshow p }=0.11, \text { correctamente clasificados } 91.82 \% \\
\text { y test de unión hat } \mathrm{p}<0.001, \text { hatsq } \mathrm{p}=0.063\end{array}$ \\
\hline
\end{tabular}




\section{Discusión}

Es interesante cómo se evidencia una correlación inversa robusta entre el porcentaje de músculo total con otros aspectos como grasa visceral, porcentaje de grasa corporal total (Figura 1), índice de masa corporal y perímetro de cintura; demostrando como al incrementarse estos últimos tiende a disminuir la cantidad de masa magra. Estos hechos inciden en la presencia de síndrome metabólico y riesgo de diabetes, como otros estudios lo han demostrado (10).

La sarcopenia se ha estudiado principalmente en personas mayores y en este estudio se muestra según criterios europeos de sarcopenia en el rango moderado en más de $50 \%$ de los pacientes menores de 60 años y grado severo en promedio de $15 \%$. Igualmente llama la atención la presencia en $60.53 \%$ de los pacientes menores de 30 años, quienes presentan una pérdida de masa muscular, compatible con criterios europeos de sarcopenia de grado moderado y grado severo en $22.37 \%$. Este hallazgo podría demostrar alteraciones nutricionales y hábitos de ejercicio, que contribuyen a la presencia de sarcopenia $(11,12)$. Además se encontró en los diferentes grupos etáreos una asociación importante de sarcopenia con problemas de sobrepeso u obesidad, incluyendo a los pacientes menores de 30 años, constituyendo el cuadro de "obesidad sarcopénica", el cual tienen que ver con los cambios de la composición corporal, asociados con el incremento de la grasa corporal y disminución del músculo esquelético, relacionados con la edad e incremento de la grasa visceral, el cual contribuye con factores de riesgo cardiometabólico (13).

Se encuentra en el análisis bivariado, factores protectores asociados a sarcopenia, como es el peso, índice de masa corporal, grasa corporal total y sin una significancia estadística la edad y la grasa visceral, consideramos que este análisis tiene limitaciones en cuanto a la validez, ya que está siendo influenciado por variables de confusión; este hecho es evi-

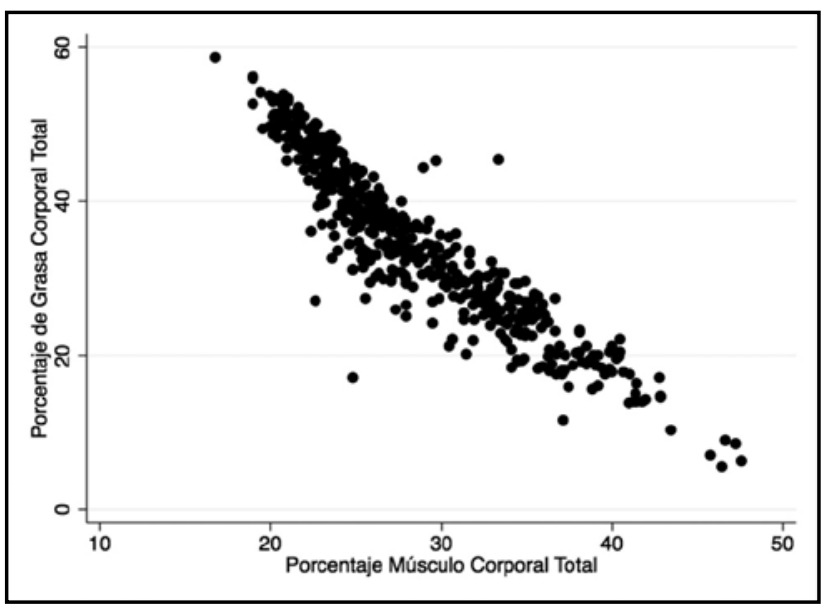

Figura 1. Relación entre grasa corporal total y porcentaje de músculo corporal total. Se encuentra una relación inversa entre el porcentaje de grasa corporal total y el porcentaje de músculo corporal total. Al determinar la correlación Spearman fue inversa entre el porcentaje de músculo total y el porcentaje de grasa corporal total y estadísticamente significativa (rho $=-0.855$ para mujeres y rho $=-0.882$ para hombres $p<0.01$ ). dente cuando se realizó al análisis multivariado donde los factores con mayor fuerza asociados a sarcopenia fueron la edad, el sexo masculino y el porcentaje de grasa visceral. Como se aprecia, ésta es una visión reduccionista del fenómeno llamado sarcopenia, ya que estudios han demostrado que la sarcopenia es multifactorial incluyendo: déficit de vitamina $\mathrm{D}(12,14)$, deficiencia de testosterona (14), actividad proinflamatoria, estrés oxidativo (15), disminución de ingesta de proteínas (12) y porcentaje de masa grasa (16).

Los hallazgos de sarcopenia moderada o incluso severa, en pacientes jóvenes menores de 30 años, podría ser la manifestación de malnutrición, donde la ingesta en nuestro medio son las dietas ricas en carbohidratos, favoreciendo déficit proteico y menor construcción de músculo. Vemos que este déficit es marcado en todos los grupos etáreos, donde se incrementa el riesgo de síndrome metabólico, diabetes y riesgo cardiovascular $(2,17)$. La presencia de sarcopenia muestra una relación directa con grasa visceral y porcentaje de grasa corporal total, este hallazgo podría sugerir mecanismos metabólicos en inclusive en edades tempranas (menores de 30 años), conformando al cuadro de obesidad sarcopénica, en donde el incremento de la grasa visceral forja un proceso, en el cual hay una pérdida de la cantidad de músculo, acumulación de grasa ectópica, generando factores como citoquinas proinflamatorias, estrés oxidativo, disfunción mitocondrial e insulino-resistencia $(13,18)$.

Estudios como el coreano de obesidad sarcopénica (KSOS), encontró sarcopenia asociado a problemas de sobrepeso u obesidad, incluyendo pacientes jóvenes con una edad media $51.9 \pm 14.6$ años, usando densitometría en donde se evidenció incremento en la grasa corporal total, grasa visceral y disminución del contenido muscular; hallazgos semejantes a lo encontrado en este estudio (19).

$\mathrm{Al}$ igual que en el hueso, el músculo alcanza un pico en su formación y desarrollo que es entre 20 y 30 años de edad, el tratar de definir sarcopenia en menores de 30 años, puede ser un factor de confusión y llevar a conclusiones fuera de contexto; sin embargo, es importante tener en cuenta que el déficit nutricional, incluyendo oligoelementos y vitamina $\mathrm{D}$, pueden originar que el pico de masa muscular en edades tempranas no se alcance, y por lo tanto los hallazgos en este estudio sean la manifestación de lo anterior, más que una pérdida de masa muscular o sarcopenia (12).

Se consideran como limitaciones de este estudio, el desconocimiento de las comorbilidades de los pacientes que pudieran tener impacto sobre la interpretación de los datos (artritis reumatoide, cáncer de seno, enfermedad pulmonar obstructiva crónica y enfermedad renal crónica); desconocimiento del grado de inactividad física para tratar de inferir otros mecanismos patológicos de la sarcopenia.

Consideramos importante plantear para futuros estudios determinar aspectos relacionados con la sarcopenia en edades tempranas, diferentes a la inactividad física, por ejemplo: inflamación, o factores liberados por miocitos, vitamina D, diabetes, entre otros (20-23). 


\section{Conclusión}

La pérdida o la falta de un adecuado desarrollo de masa muscular se evidencia desde edades tempranas, la definición de sarcopenia es limitada y consideramos que se deben realizar estudios donde se incluya población joven, con análisis multivariados donde se logren buscar factores asociados a la baja masa muscular; además plantear nueva definición de sarcopenia en donde se integren diferentes grupos etáreos.

\section{Declaración de fuentes de financiación y conflictos de intereses}

El grupo de autores declaramos, que la fuente de apoyo financiero fue por recursos propios y no tenemos conflicto de intereses

\section{Referencias}

1. Ribeiro SM, Kehayias JJ. Sarcopenia and the Analysis of Body Composition. Advances in nutrition. 2014;5(3):260-7.

2. Yu R, Leung J, Woo J. Incremental Predictive Value of Sarcopenia for Incident Fracture in an Elderly Chinese Cohort: Results From the Osteoporotic Fractures in Men (MrOs) Study. J Am Med Dir Assoc. 2014 Aug;15(8):551-8.

3. Vetrano DL, Landi F, Volpato S, Corsonello A, Meloni E, Bernabei R, et al. Association of Sarcopenia With Short- and Long-term Mortality in Older Adults Admitted to Acute Care Wards: Results From the CRIME Study. J Gerontol A Biol Sci Med Sci. 2014;69(9):1154-61

4. Xu L, Cheng X, Wang J, Cao Q, Sato T, Wang M, et al. Comparisons of bodycomposition prediction accuracy: a study of 2 bioelectric impedance consumer devices in healthy Chinese persons using DXA and MRI as criteria methods. Journal of clinical densitometry: the official journal of the International Society for Clinical Densitometry. 2011;14(4):458-64.

5. Valenzuela-Landaeta K, Rojas P, Basfi-fer K. [Nutritional assessment for cancer patient]. Nutricion hospitalaria. 2012;27(2):516-23.

6. Ozhan H, Alemdar R, Caglar O, Ordu S, Kaya A, Albayrak S, et al. Performance of bioelectrical impedance analysis in the diagnosis of metabolic syndrome. J Investig Med. 2012;60(3):587-91.

7. Nagai M, Komiya H, Mori Y, Ohta T, Kasahara Y, Ikeda Y. Estimating visceral fat area by multifrequency bioelectrical impedance. Diabetes care. 2010;33(5):1077-9.

8. Cruz-Jentoft AJ, Baeyens JP, Bauer JM, Boirie Y, Cederholm T, Landi F, et al. Sarcopenia: European consensus on definition and diagnosis: Report of the European Working Group on Sarcopenia in Older People. Age and ageing. 2010;39(4):412-23.

9. http://www.omronhealthcare.com/wp-content/uploads/hbf-510w-instructionmanual.pdf. .

10. Sanada K, Iemitsu M, Murakami H, Gando Y, Kawano H, Kawakami R, et al. Adverse effects of coexistence of sarcopenia and metabolic syndrome in Japanese women. Euro J clini nutrit. 2012; 66(10): 1093-8.

11. Laviano A, Gori C, Rianda S. Sarcopenia and nutrition. Advan food nutrit research. 2014; 71: 101-36.

12. Robinson S, Cooper C, Aihie Sayer A. Nutrition and sarcopenia: a review of the evidence and implications for preventive strategies. J aging research. 2012; 2012: $510-801$.

13. Kim TN, Choi KM. The implications of sarcopenia and sarcopenic obesity on cardiometabolic disease. J Cell Biochem. 2014; 29. doi: 10.1002/jcb.25077. [Epub ahead of print].

14. Sakuma K, Yamaguchi A. Sarcopenia and age-related endocrine function. Int J Endocrinol. 2012; 2012: 127-362.

15. Rom O, Kaisari S, Aizenbud D, Reznick AZ. Lifestyle and sarcopenia-etiology, prevention, and treatment. Rambam Maimonides Med J. 2012; 3(4): e0024.

16. Krause KE, McIntosh EI, Vallis LA. Sarcopenia and predictors of the fat free mass index in community-dwelling and assisted-living older men and women. Gait \& posture. 2012; 35(2): 180-5.

17. Kim JH, Lim S, Choi SH, Kim KM, Yoon JW, Kim KW, et al. Sarcopenia: An Independent Predictor of Mortality in Community-Dwelling Older Korean Men. J Gerontol A Biol Sci Med Sci. 2014; 69(10): 1244-52.

18. Park SH, Park JH, Park HY, Jang HJ, Kim HK, Park J, et al. Additional role of sarcopenia to waist circumference in predicting the odds of metabolic syndrome. Clin Nutr. 2014; 33(4): 668-72.

19. Kim TN, Park MS, Ryu JY, Choi HY, Hong HC, Yoo HJ, et al. Impact of Visceral Fat on Skeletal Muscle Mass and Vice Versa in a Prospective Cohort Study: The Korean Sarcopenic Obesity Study (KSOS). PloS one. 2014; 9(12): e115407.

20. Vaidya R. Obesity, sarcopenia and postmenopausal osteoporosis: An interlinked triad. J mid-life health. 2014; 5(1): 1-2.

21. Kim MK, Baek KH, Song KH, Il Kang M, Park CY, Lee WY, et al. Vitamin D deficiency is associated with sarcopenia in older Koreans, regardless of obesity: the Fourth Korea National Health and Nutrition Examination Surveys (KNHANES IV) 2009. J Clin Endocrinol Metab. 2011; 96(10): 3250-6.

22. Kim TN, Park MS, Yang SJ, Yoo HJ, Kang HJ, Song W, et al. Prevalence and determinant factors of sarcopenia in patients with type 2 diabetes: the Korean Sarcopenic Obesity Study (KSOS). Diabetes care. 2010; 33(7): 1497-9.

23. Biolo G, Cederholm T, Muscaritoli M. Muscle contractile and metabolic dysfunction is a common feature of sarcopenia of aging and chronic diseases: From sarcopenic obesity to cachexia. Clin Nutr. 2014; 33(5): 737-48. 